\title{
THE FOSSIL LAND AND FRESHWATER SNAILS OF OGGENHAUSEN (MIDDLE MIOCENE, GERMANY)
}

\author{
RODRIGO B. SALVADOR \& MICHAEL W. RASSER \\ Staatliches Museum für Naturkunde Stuttgart; Stuttgart, Germany. Rosenstein 1, 70191, Stuttgart, Germany. \\ salvador.rodrigo.b@gmail.com,michael.rasser@smns-bw.de
}

\begin{abstract}
The Middle Miocene fossil site of Oggenhausen in southern Germany was first described in the 1920's, with reports of nine gastropod species. Much later, in the 2000's, a second and coeval site was described, named Oggenhausen 2 and counting with eight gastropod species, all of them distinct from the first site (hence named Oggenhausen 1). Here we present a revision of the historical material from Oggenhausen 1 alongside new material from Oggenhausen 2. After the revision, six species are known from Oggenhausen 1 (the records of Triptychia kleini and Gastrocopta acuminata could not be confirmed) and 13 from Oggenhausen 2. The following are new records for Oggenhausen 2: Lymnaea cf. dilatata, Gyraulus applanatus, Megalotachea silvana, Palaeotachea renevieri, Granaria sp., Gastrocopta acuminata and ?Truncatellina sp. Oggenhausen 2 is interpreted as a shallow lake or an oxbow lake, formed by the river that deposited the layers of Oggenhausen 1. The freshwater snails are consistent with slow-flowing or stagnant waters, while the terrestrial species indicate that the area surrounding the lake was covered by humid vegetation.
\end{abstract}

Key words: Caenogastropoda, Gastropoda, Hygrophila, OSM, Stylommatophora.

RESUMO - O afloramento fossilífero de Oggenhausen (Mioceno Médio, sul da Alemanha) foi originalmente descrito na década de 1920 e nove espécies de gastrópodes foram encontradas nos sedimentos. Posteriormente, na década de 2000, um segundo (e contemporâneo) afloramento foi descrito como Oggenhausen 2, contando com oito espécies de gastrópodes, todas distintas do primeiro afloramento (nomeado Oggenhausen 1). O presente trabalho traz uma revisão do material histórico de Oggenhausen 1, assim como material inédito de Oggenhausen 2. Após a revisão, constatou-se a presença de seis espécies em Oggenhausen 1 (os registros de Triptychia kleini e Gastrocopta acuminata não puderam ser confirmados) e treze em Oggenhausen 2. Os registros inéditos para Oggenhausen 2 são: Lymnaea cf. dilatata, Gyraulus applanatus, Megalotachea silvana, Palaeotachea renevieri, Granaria sp., Gastrocopta acuminata e ?Truncatellina sp. Oggenhausen 2 é considerado um lago raso ou um meandro abandonado do rio que depositou as camadas de Oggenhausen 1. Os gastrópodes dulciaquícolas são consistentes com águas estagnadas ou de fluxo lento, enquanto as espécies terrestres indicam que a área ao redor do lago era coberta por uma vegetação mais fechada e úmida.

Palavras-chave: Caenogastropoda, Gastropoda, Hygrophila, OSM, Stylommatophora.

\section{INTRODUCTION}

The Middle Miocene fossil site of Oggenhausen, a village in southern Germany near to the town of Heidenheim in Baden-Württemberg state, was first described by Schlosser (1926), who dealt with the vertebrate remains. Berz \& Jooss (1927) studied the gastropods from this locality, with samples stemming from various sites around the village. They reported the following species (names as they appear in the original publication): Zonites (Aegopis) costatus costatus, Tropidomphalus (Pseudochloritis) incrassatus incrassatus, Cepaea renevieri coniuncta, $C$. silvana silvana, C. eversa larteti, Triptychia (Triptychia) grandis, Gastrocopta aff. G. acuminata acuminata, Tudorella conica conica and Melanatria escheri turrita.

Böttcher et al. (2009) described a second coeval site in Oggenhausen, named "Oggenhausen 2". Henceforth, the first site described by Schlosser (1926) and Berz \& Jooss
(1927) was referred to as "Oggenhausen 1". Böttcher et al. (2009) reported eight gastropod species from this second site (Galba dupuyiana, Radix sp., Planorbarius cornu, Anisus sp., Gastrocopta cf. G. nouletiana, Discus pleuradrus, Leucochroopsis sp. and Cepaea sp.), all belonging to the Pulmonata.

Here we present a revision of the historical material of Berz \& Jooss (1927), providing figures for the first time, and revising the material from Oggenhausen 2, also introducing new material that was housed in the collection of the Staatliches Museum für Naturkunde Stuttgart, Stuttgart, Germany. Moreover, several of the species listed here represent new distribution records for Oggenhausen.

\section{GEOLOGICAL SETTING}

Oggenhausen as a whole is located at the northern margin of the North Alpine Foreland Basin (Figure 1) and its 


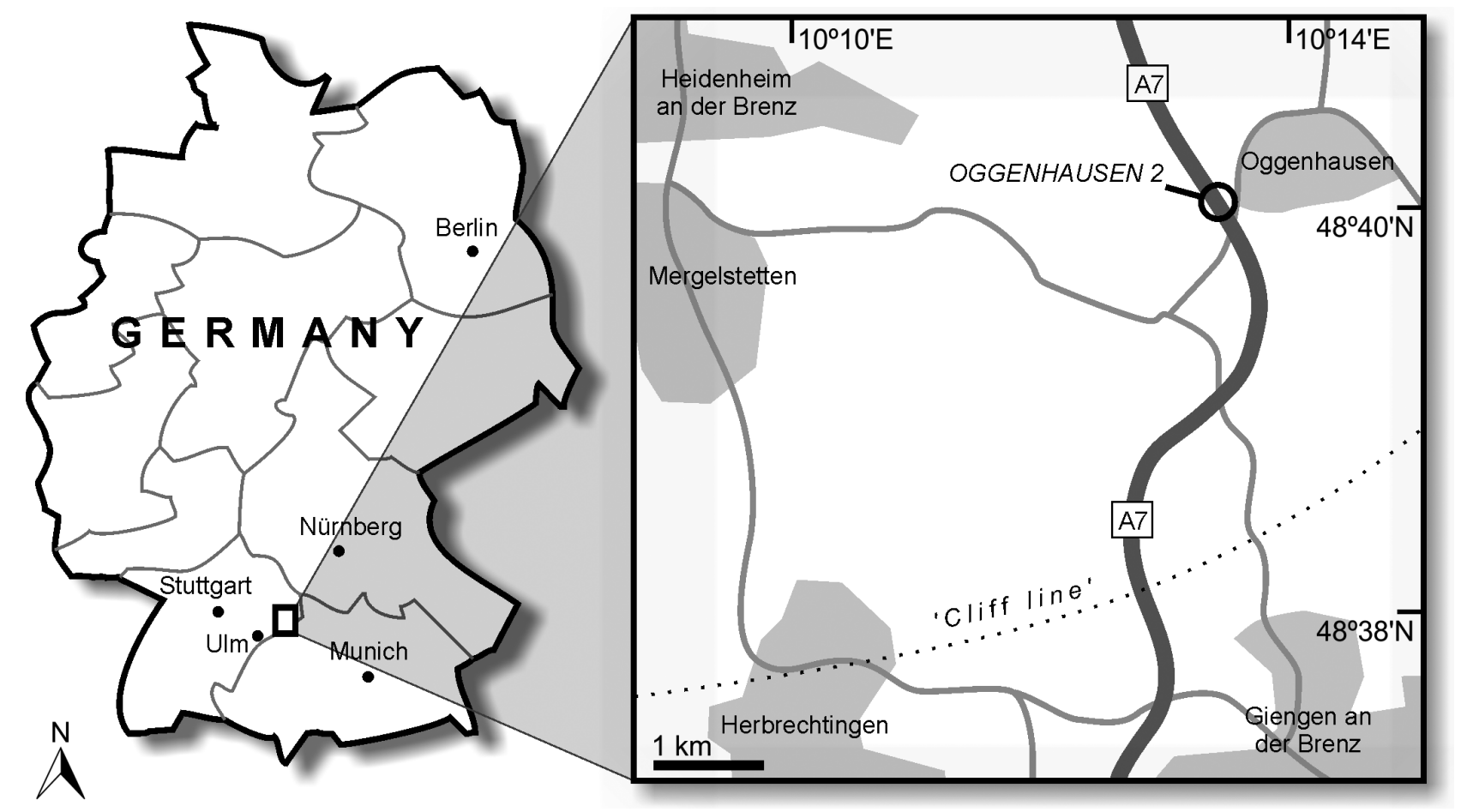

Figure 1. Map of Germany showing the location of the fossil outcrops of Oggenhausen 2 (modified after Böttcher et al., 2009). The exact location of the outcrops of Oggenhausen 1 is unknown.

outcrops belong to the Upper Freshwater Molasse ("Obere Süßwassermolasse", OSM) stratigraphic unit (more specifically to the so-called Silvana Beds, or "Silvanaschichten"). The site Oggenhausen 1 ("Oggenhausen N' Ulm" in the original; Schlosser, 1926) contained a handful of distinct small outcrops, explored by Berz \& Jooss (1927). Unfortunately, the precise location of these outcrops is now untraceable. The fossil-bearing sediments from this locality were referred to as "Oggenhausen-Sande" (Böttcher et al., 2009).

Later, in 1980, another fossil site was found in the Oggenhausen area (called "Oggenhausen 2" by Böttcher et al., 2009; Figure 1). This outcrop lies $\sim 1.5 \mathrm{~km}$ to the south of Oggenhausen 1 (topographically deeper than that locality by $c a .30 \mathrm{~m}$, but not confirmedly stratigraphically older) and was artificially created by highway construction (Heizmann, 1983; Böttcher et al., 2009). There were two main facies outcropping in the locality, a basal limestone one and a marl/ clay one, both bearing fossil gastropods (for more details, see Böttcher et al., 2009).

Biostratigraphic correlation of Oggenhausen 2 is possible due to the presence of small mammalian teeth, correlating the deposits to the middle of the European Mammal Neogene zone MN 5 (Böttcher et al., 2009; Reichenbacher et al., 2013). However, the stratigraphic relation between the sediments of Oggenhausen 1 and 2 remains unclear, due to the different mammalian faunas found in each locality (see Böttcher $\mathrm{et} \mathrm{al}$., 2009 for more details). Nevertheless, Böttcher et al. (2009) considers that Oggenhausen 1 also belong to the middle of the MN 5 zone (this zone goes from $c a .17$ to $16 \mathrm{Ma}$; Reichenbacher et al., 2013).

\section{MATERIAL AND METHODS}

The original material of Berz \& Jooss (1927) from Oggenhausen 1 and of Böttcher et al. (2009; sample of $600 \mathrm{~kg}$, screenwashed with a minimum mesh size of 0.5 $\mathrm{mm}$ ) from Oggenhausen 2 are housed in the collection of the Staatliches Museum für Naturkunde Stuttgart (SMNS), Stuttgart, Germany. The collection also housed additional material from Oggenhausen 2 that was not examined by Böttcher et al. (2009) and that is studied here for the first time.

Unfortunately two of the original specimens from Berz \& Jooss (1927) could not be found, namely the single specimens known of Triptychia (Triptychia) grandis (Klein, 1846) and Gastrocopta aff. G. acuminata acuminata (Klein, 1846) (Berz \& Jooss, 1927: species nrs. 6 and 7, respectively). Triptychia grandis has been recently renamed as Triptychia kleini Schnabel, 2006.

Preservation is poor for the largest part of the gastropod material, although reasonably well-preserved specimens could be found for nearly all species. Some taxa are known only from fragmentary specimens and their identification must remain tentative. Gastropod classification follows Bouchet $\&$ Rocroi (2005), with further modifications of Nordsieck (2014) regarding the European fossil land snail fauna. All species are figured and information on their diagnostic features is provided in the "Remarks" section of each species. Specimens were measured either with a digital caliper or with the aid of computer software (Leica Application Suite [LAS] v.3.8.0). The following abbreviations for shell measures are 
used throughout the text: $\mathbf{H}$, shell height; $\mathbf{D}$, = shell greatest width (diameter).

\section{SYSTEMATIC PALEONTOLOGY}

Clade CAENOGASTROPODA Cox, 1960

Superfamily CERITHIOIDEA Fleming, 1822

Family PACHYCHILIDAE P. Fischer \& Crosse, 1822

Tinnyea Hantken, 1887

Type species. Tinnyea vasarhelyii Hantken, 1887.

Tinnyea lauraea (Mathéron, 1842)

(Figures 2A-B)

1842 Melania lauraea Mathéron, p. 291, pl. 36, figs. 23-24. 1927 Melanatria escheri turrita Brongniart: Berz \& Jooss, p. 206. 1953 Brotia (Tinnyea) escheri (Brongniart): Papp, p. 128, pl. 3, fig. 29.

1976 Brotia (Tinnyea) escheri (Brongniart): Schlickum, p. 4, pl. 1, fig. 9 .

2000 Brotia (Tinnyea) escheri (Brongniart): Mikuz \& Pavsic, p. 44 , pl.1, figs. 1-8.

2014 Tinnyea lauraea (Mathéron): Neubauer et al., supplementary material 1.

2015b Tinnyea lauraea (Mathéron): Salvador et al., p. 203, figs. 2A-B.

Material examined. Oggenhausen 1: SMNS 68388 (one spc.). Dimensions. SMNS 68388 (fragmentary specimen): $\mathrm{H}=13.7 \mathrm{~mm}$.

Remarks. The species can be identified by its turreted shell and characteristic teleoconch sculpture (very strong opisthocline and slightly curved axial ribs on first whorls and both axial ribs and spiral cords on remaining whorls). The name Tinnyea escheri Brongniart, 1822 was considered a nomen nudum (Kadolsky, 1995; Kowalke, 2004), for which the earliest valid name available is T. lauraea.

Berz \& Jooss (1927) identified this single specimen as belonging to the subspecies Tinnyea lauraea turrita, which would differ from the nominate subspecies by its simple and straight ribs, instead of spiny or knobby ones (Kókay, 2006). Harzhauser et al. (2002), however, state that the species has a great morphological plasticity (as Recent Pachychilidae do), which led to the description of several superfluous subspecies. T. lauraea is known from the Oligocene-Pliocene of Central Europe (Harzhauser et al., 2002), but such large time span suggests that the species is actually an unresolved species complex (Kadolsky, 1995).

Tinnyea has been commonly considered a subgenus of Brotia H. Adams, 1866 (Pacaud \& Le Renard, 1995). Harzhauser et al. (2002), however, consider Tinnyea a separate genus based on protoconch development and sculpture. This classification is followed here.

Superfamily LITTORINOIDEA Children, 1843 Family POMATIIDAE Newton, 1891
Pomatias Studer, 1789

Type species. Nerita elegans Müller, 1774.

Pomatias conicus (Klein, 1853)

(Figure 2C)

1853 Cyclostoma conicum Klein, p. 217, pl. 5, fig. 14. 1823 Tudorella conica conica (Klein): Wenz, p. 1820.

1927 Tudorella conica conica (Klein): Berz \& Jooss, p. 206. 1953 Pomatias conicus conicus (Klein): Papp \& Thenius, p. 21, pl. 3, fig. 7 .

1976 Tudorella conica (Klein): Schlickum, p. 3, pl. 1, fig. 2-3. 1981 Pomatias conica (Klein) [sic]: Lueger, p. 10, pl. 1, figs. 11-12, pl. 6, fig. 3 .

2006 Pomatias conica (Klein) [sic]: Kókay, p. 33, pl. 4, fig. 4. 2002 Pomatias conicus (Klein): Harzhauser \& Kowalke, p. 70, pl. 10, figs. 6-8.

2015a Pomatias conicus (Klein): Salvador et al., p. 253, fig. 3A. 2004 Pomatias conicum (Klein) [sic]: Harzhauser \& Binder, p. 7, pl.1, figs. 7-11.

Material examined. Oggenhausen 1: SMNS 68038 (one spc.), 68041 (one spc.).

Dimensions. SMNS 68041 (fragmentary specimen): $\mathrm{H}=5.9$ $\mathrm{mm}, \mathrm{D}=5.7 \mathrm{~mm}$.

Remarks. The species can be identified by its trochiform shell, with greatly rounded whorls and the teleoconch sculpture (reticulated, with stronger spiral ribs and weaker axial ribs). Pomatias conicus is known from the Middle to Late Miocene of Central Europe, being commonly found in the Silvana-beds ("Silvanaschichten") of Germany and Switzerland (Klein, 1853; Harzhauser \& Binder, 2004; Salvador et al., 2015a).

Clade PULMONATA Cuvier, 1814

Clade HYGROPHILA Férussac, 1822

Superfamily LYMNAEOIDEA Rafinesque, 1815

Family LYMNAEIDAE Rafinesque, 1815

Galba Schrank, 1803

Type species. Buccinum truncatulum Müller, 1774.

Galba dupuyiana (Noulet, 1854)

(Figures 2D-E)

1854 Limnea Dupuyiana Noulet, p. 108.

1923 Radix (Radix) dupuyana (Noulet) [sic]: Wenz, p. 1242. 2000 Galba (Galba) dupuyiana (Noulet): Fischer, p. 135, fig. 4.

2006 Galba dupuyana (Noulet) [sic]: Kókay, p. 50, pl. 16, figs. 14-16.

2009 Galba dupuyiana (Noulet): Böttcher et al., p. 239, figs. $2 / 1-2 / 2$.

2014 Galba dupuyiana (Noulet): Neubauer et al., supplementary material 1. 
2014 Galba dupuyiana (Noulet): Salvador \& Rasser, p. 188, figs. 1-7.

2016 Galba dupuyiana (Noulet): Salvador et al., p. 133, fig. 2E. Material examined. Oggenhausen 2: SMNS 68650 (one spc.), 68651 (one spc.). 101672 (>50 spc.).

Dimensions. $4-4 \frac{1}{2}$ whorls, $\mathrm{H}=5.0-6.0 \mathrm{~mm}, \mathrm{D}=2.5-3.0 \mathrm{~mm}$. Remarks. As remarked by Böttcher et al. (2009: figs. 2/1$2 / 2$ ), there are two extreme shell forms in Oggenhausen 2, a more slender one, with a more deeply marked suture (Figure 2D), and a broader one (Figure 2E). Transitional forms from slender to broad can also be found in the material (Böttcher $e t$ al., 2009). The same variability was observed in the abundant G. dupuyiana specimens from Sandelzhausen (Salvador \& Rasser, 2014). This species is known from the Middle Miocene of Central and West Europe (Fischer, 2000; Kókay, 2006).

\section{Lymnaea Lamarck, 1799}

Type species. Helix stagnalis Linnæus, 1758.

Lymnaea cf. L. dilatata (Noulet, 1854)

(Figure 2F)

2009 Radix sp.: Böttcher et al., p. 239, fig. 2/3.

Material examined. Oggenhausen 2: SMNS 68652 (one spc.).

Dimensions. SMNS 68652: $\mathrm{H}=7.2 \mathrm{~mm}$.

Remarks. Despite being a juvenile or fragmentary specimen, it is very reminiscent of Lymnaea dilatata, due to its proportionately small and acuminated spire, with quickly growing whorls. This species is known from the entire Miocene of Central and West Europe (Fischer, 2000; Binder, 2004; Kókay, 2006).

Superfamily PLANORBOIDEA Rafinesque, 1815 Family PLANORBIDAE Rafinesque, 1815

Gyraulus Charpentier, 1837

Type species. Planorbis albus Müller, 1774.

Gyraulus applanatus (Thomä, 1845)

(Figures 2G-H)

1845 Planorbis applanatus Thomä, p. 150.

1851 Planorbis dealbatus Braun, p. 1134.

1923 Gyraulus (Gyraulus) trochiformis applanatus (Thomä): Wenz, p. 1579.

1923 Gyraulus (Gyraulus) trochiformis dealbatus (Braun): Wenz, p. 1591.

1964 Gyraulus trochiformis dealbatus (Braun): Schlickum, p. 15 , pl. 2, fig. 35 .

1970a Gyraulus trochiformis applanatus (Thomä): Schlickum, p. 148 , pl. 10, fig. 6 .

1970b Gyraulus trochiformis applanatus (Thomä): Schlickum, p. 180.
1995 Gyraulus dealbatus (Braun): Kadolsky, p. 40, fig. 47. 1989 Gyraulus trochiformis dealbatus (Braun): Reichenbacher, 172 , pl. 1, fig. 11.

2004 Gyraulus dealbatus (Braun): Binder, p. 193, pl. 2, figs. 1a-c. 2005 Gyraulus applanatus (Thomä): Kowalke \& Reichenbacher, p. 631, figs. 9.1-9.3.

2006 Gyraulus applanatus (Thomä): Kókay, p. 56, pl. 19, figs. 13-14.

2006 Gyraulus trochiformis dealbatus (Braun): Kókay, p. 57, pl. 19, fig. 15 .

2009 Anisus sp.: Böttcher et al., p. 239, figs. 2/7-2/9.

2014 Gyraulus applanatus (Thomä): Neubauer et al., supplementary material 1.

2014 Gyraulus dealbatus (Braun): Neubauer et al., supplementary material 1.

2014 Gyraulus dealbatus (Braun): Salvador \& Rasser, p. 192, figs. 16-23.

2015b Gyraulus applanatus (Thomae): Salvador et al., p. 205, figs. 2H-I.

2016 Gyraulus applanatus (Thomae): Salvador et al., p. 134, figs. 2K-M.

Material examined. Oggenhausen 2: SMNS 68654 (three spc.), 101379 (42 spc.), 101380 (40 spc.), 101673 (>50 spc.).

Dimensions. $3 \frac{1}{4}-3 \frac{1}{1} 2$ whorls, $H=1.0-1.3 \mathrm{~mm}, \mathrm{D}=3.0$ $-4.0 \mathrm{~mm}$.

Remarks. The specimens identified as Anisus sp. by Böttcher et al. (2009) belongs to the morphologically variable species Gyraulus applanatus. The more flattened and stronger keeled forms indeed show a remarkable resemblance to species of Anisus Studer, 1820, but are recognizable as Gyraulus by their protoconch sculpture (with numerous fine spiral striae) and low number of rapidly growing whorls. Species of Anisus also present a larger number of whorls and a strongly angulated profile.

Gyraulus applanatus is recorded from the Early and Middle Miocene of Germany, being known for a great deal of morphological variation (Kowalke \& Reichenbacher, 2005; Salvador \& Rasser, 2014). This variability is also seen in Oggenhausen 2, from more rounded shells to more flattened ones, with all intermediate forms in between.

Planorbarius Duméril, 1805

Type species. Helix cornea Linnaeus, 1758.

Planorbarius mantelli (Dunker, 1848)

(Figures 2I-J)

1848 Planorbis Mantelli Dunker, p. 159, pl. 21, figs. 27-29. 1966 Planorbarius cornu (Brongniart): Schlickum, p. 326, pl. 13, fig. 27.

1970a Planorbarius cornu (Brongniart): Schlickum, p. 149, pl. 10, fig. 7.

1989 Planorbarius cornu (Brongniart): Reichenbacher, $\mathrm{p}$. 172, pl. 1, fig. 10.

2004 Planorbarius cornu (Brongniart): Binder, p. 193, pl. 2, figs. 2-3. 
2006 Planorbarius cornu cornu (Brongniart): Kókay, p. 58, pl. 20, fig. 6 .

2009 Planorbarius cornu (Brongniart): Böttcher et al., p. 239, figs. 2/4-2/6.

2014 Planorbarius cornu (Brongniart): Salvador \& Rasser, p. 193 , figs. $26-28$.

2014 Planorbarius mantelli (Dunker): Harzhauser et al., p. 15, pl. 3, figs. 5, 7-13, 15-16.

2014 Planorbarius mantelli (Dunker): Neubauer et al., supplementary material 1.

2015a Planorbarius cornu (Brongniart): Salvador et al., p. 256, figs. $3 \mathrm{G}-\mathrm{H}$.

2015b Planorbarius cornu (Brongniart): Salvador et al., p. 205, fig. 2J.

2016 Planorbarius cornu (Brongniart): Salvador et al., 135, figs. 2P-Q.

Material examined. Oggenhausen 2: SMNS 68653 (one spc.), 101670 (20 spc.).

Dimensions. SMNS 68653 (juvenile specimen): $H=3.9$ $\mathrm{mm}, \mathrm{D}=7.7 \mathrm{~mm}$.

Remarks. Despite only juveniles and fragmentary specimens being preserved, the shape and unique sculpture of the shell allow identification as Planorbarius mantelli. The protoconch sculpture (see Böttcher et al., 2009: fig. 2/6) consists of spiral lines composed of regularly disposed circular pits; teleoconch is sculptured by spiral striae on first $\sim 1 \frac{1}{2}$ whorl and smooth on the remaining whorls (except for well-marked growth lines).

This species is known from the late Early Miocene to the Late Miocene of Central Europe (Harzhauser et al., 2014). Specimens of Planorbarius mantelli from these ages and regions are usually referred to as $P$. соrnu (Brongniart, 1810) in the literature. Planorbarius cornu is a form recorded from the Late Oligocene to Early Miocene (Harzhauser et al., 2014) and very hard to diagnose from $P$. mantelli. This species complex is in urgent need of a thorough revision.

Clade STYLOMMATOPHORA A. Schmidt, 1855

Superfamily HELICOIDEA Rafinesque, 1815

Family HELICIDAE Rafinesque, 1815

Megalotachea Pfeffer, 1929

Type species. Helix turonensis Deshayes, 1831.

Megalotachea silvana (Klein, 1853)

(Figures 2K-M)

1853 Helix silvana Klein, p. 205, pl. 5, fig. 2.

1923 Cepaea silvana Silvana (Klein): Wenz, p. 667.

1927 Cepaea eversa larteti (Boissy): Berz \& Jooss, p. 205.

1927 Cepaea sylvana sylvana (Klein) [sic]: Berz \& Jooss, p. 204.

1976 Cepaea silvana Silvana (Klein): Schlickum, p. 17, pl. 4, figs. 62-63.

1989 Cepaea silvana Silvana (Klein): Reichenbacher, p. 165, pl. 2, figs. 17-19.
2006 Cepaea silvana (Klein): Kókay, p. 93, pl. 36, figs. 2-3. 2009 Cepaea sp.: Böttcher, et al., p. 241, fig. 2/15.

2015a Megalotachea silvana (Klein): Salvador et al., p. 261, figs. 4C-D.

2015b Megalotachea silvana (Klein): Salvador et al., p. 208, figs. 2U-W.

2016 Megalotachea silvana (Klein): Salvador et al., p. 137, figs. 3A-C.

Material examined. Oggenhausen 1: SMNS 66796 (four spc.), 66814 (two spc.), 66849 (five spc.), 66850 (two spc.), 66876 (one spc.), 101668 (one spc.). Oggenhausen 2: SMNS 68658 (one spc.), 101373 (one spc.), 101374 (six spc.), 101375 (two spc.).

Dimensions. $4-4 \frac{1}{2}$ whorls, $H=13.5-19.1 \mathrm{~mm}, \mathrm{D}=18.5$ $-24.5 \mathrm{~mm}$.

Remarks. Megalotachea silvana is distinguished from its congeners by its smaller size, more flattened shell profile and by the presence of a faint keel. On four specimens (two of each lot: SMNS 101374 and 101375) it is possible to observe under UV light a trifasciate pattern of colored spiral bands. A similar pattern, albeit with narrower bands, was seen on one of the Palaeotachea renevieri specimens (see below). This trifasciate pattern occurs in Recent helicids (e.g. Welter-Schultes, 2012) and was found to be the commonest one in fossils of M. sylvestrina (Schlotheim, 1820) from Poland (Górka, 2008).

Berz \& Jooss (1927) identified a single and slightly larger specimen of Megalotachea silvana (SMNS 66876) as M. eversa Deshayes, 1851 , which is a morphologically very similar species, diagnosed mainly by its much larger size. However, this specimen from Oggenhausen 1, as well as two more somewhat larger shells from Oggenhausen 2 (one specimen from each lot: SMNS 101374 and 101375), are still much smaller than the typical M. eversa and conchologically indistinguishable from M. silvana. In their paper, Berz \& Jooss (1927) mention more than one specimen of M. eversa from different places in Oggenhausen 1, but only the single specimen mentioned above was found.

Palaeotachea Jooss, 1912

Type species. Helix crepidostoma Sandberger, 1872

Palaeotachea renevieri (Maillard, 1892) (Figures 2N-P)

1892 Helix (Macularia) Renevieri Maillard, p. 43, pl. 3, fig. 18. 1923 Cepaea renevieri (Maillard): Wenz, p. 652.

1927 Cepaea renevieri coniuncta Berz \& Jooss, p. 203, fig. 1. 1954 Cepaea cf. renevieri (Maillard): Zöbelein, p. 156.

2015a Palaeotachea renevieri (Maillard): Salvador et al., p. 261, figs. 4A-B.

2015b Palaeotachea renevieri (Maillard): Salvador et al., p. 208, figs. 2R-T.

2016 Palaeotachea renevieri (Maillard): Salvador et al., p. 137, figs. 3D-F. 
Material examined. Oggenhausen 1: SMNS 66453 (one spc.), 66456 (three spc.), 66457 (two spc.), 66840 (three spc.), 101369 (two spc.), 101370 (three spc.), 101371 (four spc.), 101372 (one spc.). Oggenhausen 2: SMNS 101368 (one spc.). Dimensions. 4 whorls, $\mathrm{H}=10.0-12.0 \mathrm{~mm}, \mathrm{D}=14.9-17.4 \mathrm{~mm}$. Remarks. Palaeotachea renevieri can be easily identified by its small size, small circular aperture and somewhat flattened shell profile with a faint keel. On two specimens (SMNS 101368 and 101372) it is possible to observe vestiges of colored parallel spiral bands under UV light. The former shows a trifasciate pattern with very fine spiral bands: the topmost one on the middle to upper portion of the whorl, the other two regularly spaced on the basal portion of whorl. The latter exhibits a fourth band, positioned on the upper portion of the whorl, above all others.

Berz \& Jooss (1927) described a new variety of this species with the poorly preserved material from Oggenhausen, namely Palaeotachea renevieri coniuncta, diagnosed by a slightly larger size, more flattened profile and a smaller and rounder aperture. Furthermore, Berz \& Jooss (1927: p. 203) state that the two forms are connected by many intermediates and are hard to distinguish. These characters are clearly insufficient to diagnose two subspecies. Thus, we treat $P$. renevieri coniuncta as a synonym of the nominate $P$. renevieri. The species is known from the Middle and Late Miocene of southern Germany and Switzerland (Wenz, 1923; Zöbelein, 1954).

Pseudochloritis Boettger, 1909

Type species. Helix incrassata Klein, 1853.

\section{Pseudochloritis incrassata (Klein, 1853)}

(Figures 2Q-S)

1846 Helix inflexa Klein, p. 71, pl. 1, fig. 12 [non von Zieten, $1832]$.

1853 Helix incrassata Klein, p. 208, pl. 5, fig. 6.

1923 Tropidomphalus (Pseudochloritis) incrasstus incrassatus (Klein): Wenz, p. 510.

1927 Tropidomphalus (Pseudochloritis) incrasstus incrassatus (Klein): Berz \& Jooss, p. 202.

1976 Tropidomphalus (Pseudochloritis) incrasstus incrassatus (Klein): Schlickum, p. 16, pl. 4, fig. 56.

2006 Tropidomphalus (Pseudochloritis) cf. incrassatus (Klein): Kókay, p. 90, pl. 34, figs. 12-14.

2008 Pseudochloritis incrassata (Klein): Binder, p. 172, pl. 3, figs. 2-4, pl. 6, fig. 2.

2013 Pseudochloritis incrassata (Klein): Rasser et al., p. 434, pl. 4, fig. 12.

2014 Pseudochloritis incrassata (Klein): Harzhauser et al., p. 35, pl. 12, figs. 9-16, 19-24.

2015 Pseudochloritis incrassata (Klein): Höltke \& Rasser, p. 1, figs. 4.3, 5.1, 6.11-6.12.

2015a Pseudochloritis incrassata (Klein): Salvador et al., p. 261, figs. 4E-G.

2015b Pseudochloritis incrassata (Klein): Salvador et al., p. 207 , figs. 3A-C.
Material examined. Oggenhausen 1: SMNS 66213 (four spc.), 66657 (one spc.). Oggenhausen 2: SMNS 101377 (one spc.).

Dimensions. $4 \frac{1}{2}-4 \frac{3}{4}$ whorls, $H=14.6-18.6 \mathrm{~mm}, \mathrm{D}=$ $22.9-26.6 \mathrm{~mm}$.

Remarks. This species can be identified by its depressed flattened spire, sculpture pattern, and the lightly bent body whorl (with a conspicuous constriction right before the aperture; “extralabial depression" sensu Binder, 2008). The protoconch sculpture pattern consists of fine striae, while the teleoconch pattern consists of coarse growth lines and regularly spaced and very shallow round pits

On one specimen (SMNS 101377) it is possible to observe vestiges of two parallel spiral bands on the middle portion of the body whorl under UV light. This bifasciate color pattern differs from the observations of Górka (2008: fig. 3), which reports two additional bands. Thus, this possibly indicates some degree of intraspecific variability, as seen in some recent helicids.

Pseudochloritis incrassata is known from the Middle Miocene of Poland (Górka, 2008), Austria, many localities in Germany (Binder, 2008) and perhaps Hungary (Kókay, 2006). Despite being traditionally placed in the Elonidae (e.g. Nordsieck, 2014), a recent revision (Höltke \& Rasser, 2015) deems the genus to be more closely related to the helicid subfamily Ariantinae.

\section{Family HYGROMIIDAE Tryon, 1866}

Urticicola Lindholm, 1927

Type species. Helix umbrosa Pfeiffer, 1828.

Urticicola perchtae Salvador, 2013

(Figure 2T)

2009 Hygromiidae gen. indet. sp. 2: Moser et al., 2009, p. 50. 2009 Leucochroopsis sp.: Böttcher et al., p. 241, figs. 13-14. 2013 Urticicola perchtae Salvador, p. 168, figs. 28-32.

2016 Urticicola perchtae Salvador: Salvador et al., p. 138, figs. 3H-I.

Material examined. Oggenhausen 2: SMNS 68657 (one spc.), 101378 (one spc.).

Dimensions. SMNS 68657: $\mathrm{H}=3.4 \mathrm{~mm}, \mathrm{D}=4.5 \mathrm{~mm}$.

Remarks. The following characters allow the identification as Urticicola perchtae: whorl profile with a smooth but prominent keel-like angulation; depressed spire; teleoconch sculptured by regularly distributed and vertically elongated fine scales. As already remarked by Salvador (2013), the specimen from Oggenhausen 2 identified as Leucochroopsis sp. by Böttcher et al. (2009) actually belong to Urticicola perchtae. This species is presently known only from other two MN 5 localities, Sandelzhausen (type locality; Salvador, 2013) and Riedensheim (Salvador et al., 2016), both in southeastern Germany. 
Superfamily PUNCTOIDEA Morse, 1864

Family DISCIDAE Thiele, 1931 (1866)

Discus Fitzinger, 1833

Type species. Helix ruderata Hartmann, 1821.

Discus pleuradrus (Bourguignat, 1881)

(Figure 2U)

1881 Helix pleuradra Bourguignat, p. 53, pl. 3, figs. 67-72. 1923 Gonyodiscus (Gonyodiscus) pleuradra pleuradra (Bourguignat): Wenz, p. 341.

1942 Gonyodiscus (Gonyodiscus) pleuradra pleuradra (Bourguignat): Wenz \& Edlauer, p. 93.

1967 Discus (Discus) pleuradrus (Bourguignat): Schütt, p. 213, fig. 16.

1976 Discus (Discus) pleuradrus (Bourguignat): Schlickum, p. 12, pl. 2, fig. 37.

1981 Discus (Discus) pleuradrus (Bourguignat): Lueger, p. 40, pl. 4, figs. 6-7.

2000 Discus (Discus) pleuradra (Bourguignat) [sic]: Fischer, p. 145 , fig. 21.

2009 Discus pleuradrus (Bourguignat): Böttcher et al., p. 239, figs. 2.10-2.11.

2013 Discus pleuradrus (Bourguignat): Rasser et al., p. 439. 2014 Discus pleuradrus (Bourguignat): Harzhauser et al., p. 29, pl. 9, figs. 8-13.

2014 Discus pleuradrus (Bourguignat): Salvador \& Rasser, p. 195, figs. 32-33.

2015a Discus pleuradrus (Bourguignat): Salvador et al., p. 264, figs. 4U-W.

2016 Discus pleuradrus (Bourguignat): Salvador et al., p. 140 , figs. 3M-N.

Material examined. Oggenhausen 2: SMNS 68655 (one spc.), 101671 (five spc.).

Dimensions. SMNS 68655: $\mathrm{H}=2.0 \mathrm{~mm}, \mathrm{D}=3.5 \mathrm{~mm}$.

Remarks. Discus pleuradrus is easily identified by its discoid shell, with a step-like spire, a wide umbilicus and a lightly marked keel on the median-upper portion of the whorl, and by its shell sculpture. The protoconch is smooth and the teleoconch is sculptured by strong prosocline ribs. The species is known from the entire Miocene of Central and West Europe (Böttcher $e t$ al., 2009). Nevertheless, Early and Late Miocene records could represent different species, as argued by Moser et al. (2009). Thus, D. pleuradrus could be restricted to the Middle Miocene of France, southern Germany, and Austria (Harzhauser et al., 2014).

Superfamily PUPILLOIDEA Turton, 1831 Family CHONDRINIDAE Steenberg, 1925

Granaria Held, 1838

Type species. Pupa frumentum Draparnaud, 1801.

Granaria sp.

(Figure 2V)
Material examined. Oggenhausen 2: SMNS 101367 (two spc.), 101669 (three spc.).

Dimensions. SMNS 101367 (fragmentary specimen): $\mathrm{H}=1.7 \mathrm{~mm}, \mathrm{D}=1.4 \mathrm{~mm}$.

Remarks. These broken and badly preserved shell apexes can be attributed to the genus Granaria by their very characteristic conical shape and whorl profile. In one of the specimens, the teleoconch sculpture can be seen, consisting of very fine prosocline ribs, regularly distributed and spaced from each other by circa twice the rib's width. Nevertheless, identification beyond genus level remains impossible, since the apertural barriers are the most important diagnostic features in the family (Gittenberger, 1973; Höltke \& Rasser, 2013). Granaria is a well-documented genus in the Central European Miocene (Höltke \& Rasser, 2013).

Family GASTROCOPTIDAE Pilsbry, 1918

Gastrocopta Wollaston, 1878

Type species. Pupa acarus Benson, 1856.

Gastrocopta acuminata (Klein, 1846)

(Figure 2W)

1846 Pupa acuminata Klein, p. 75, pl. 1, fig. 19.

1923 Gastrocopta (Albinula) acuminata acuminata (Klein): Wenz, p. 916.

1999 Gastrocopta acuminata (Klein): Stworzewicz, p. 161, figs. 56-58).

2000 Gastrocopta (Albinula) acuminata (Klein): Manganelli \& Giusti, p. 60, pl. 1, figs. 1-6, pl. 2, figs. 1-7.

2015 Gastrocopta acuminata (Klein): Salvador, p. 42, fig. 7. 2016 Gastrocopta acuminata (Klein): Salvador et al., p. 140 , fig. $3 \mathrm{P}$.

Material examined. Oggenhausen 2: SMNS 101675 (two spc.). Dimensions. SMNS 101675: $\mathrm{H}=2.6 \mathrm{~mm}$.

Remarks. Gastrocopta acuminata is diagnosed by its larger size, when compared with coeval congeners, and by the shape of its bifid anguloparietal lamella. It is known from the Middle Miocene to Late Pliocene (and maybe Early Pleistocene) of Europe (Stworzewicz, 1999; Manganelli \& Giusti, 2000). Unfortunately, the report of $G$. aff. acuminata of Berz \& Jooss (1927: p. 206) for Oggenhausen 1 could not be confirmed, as the material seems to be missing.

\section{Gastrocopta nouletiana (Dupuy, 1850)}

(Figure 2X)

1850 Pupa Nouletiana Dupuy, p. 309, pl. 15, fig. 6.

1923 Gastrocopta (Sinalbinula) nouletiana nouletiana (Dupuy): Wenz, p. 930.

1999 Gastrocopta nouletiana (Dupuy): Stworzewicz, p. 163, figs. 59-61.

2009 Gastrocopta cf. nouletiana (Dupuy): Böttcher et al., p. 241 , fig. $2 / 12$.

2015 Gastrocopta nouletiana (Dupuy): Salvador, p. 43, figs. 8-11. 


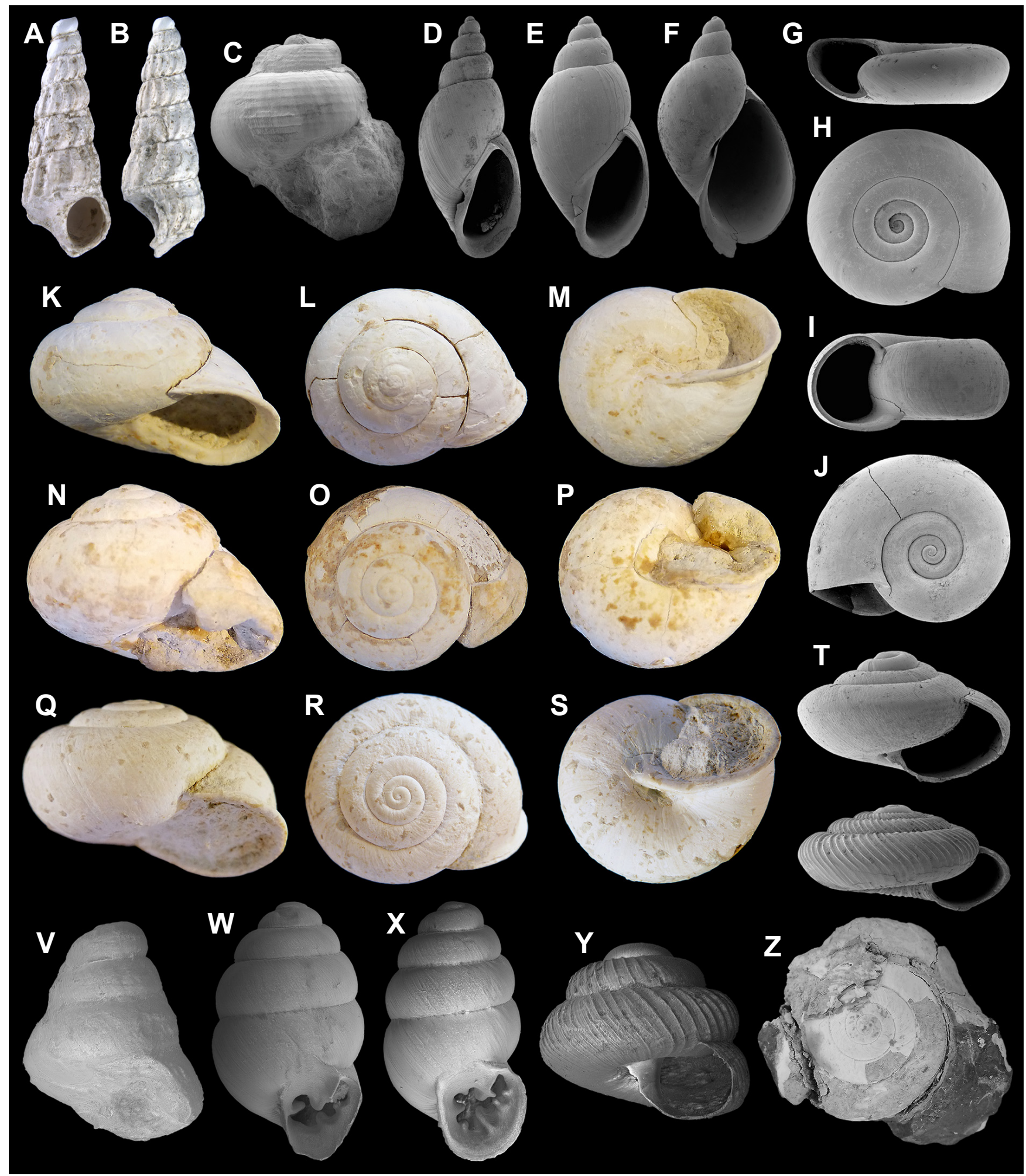

Figure 2. A-B, Tinnyea escheri, juvenile or fragmentary (SMNS 68388; $\mathrm{H}=13.7 \mathrm{~mm}$ ). C, Pomatias conicus, juvenile or fragmentary (SMNS 68041; H = $5.9 \mathrm{~mm}, \mathrm{D}=5.7 \mathrm{~mm}$ ). D, Galba dupuyiana, slender form (SMNS 68651; H = $6.5 \mathrm{~mm}$ ). E, Galba dupuyiana, broad form (SMNS $68650 ; \mathrm{H}=6.5 \mathrm{~mm}$ ). F, Lymnaea cf. dilatata, juvenile or fragmentary (SMNS 68652; $\mathrm{H}=7.2 \mathrm{~mm}$ ). G-H, Gyraulus applanatus (SMNS 68654; $\mathrm{D}=6.0 \mathrm{~mm}$ ). I-J, Planorbarius mantelli (SMNS 68653; H = $3.9 \mathrm{~mm}, \mathrm{D}=7.7 \mathrm{~mm}) . \mathrm{K}-\mathbf{M}$, Palaeotachea renevieri (SMNS 101368; D = 16.4 mm). N-P, Megalotachea silvana (SMNS 101374; D = $21.1 \mathrm{~mm}$ ). Q-S, Pseudochloritis incrassata (SMNS 66213; D = 22.9 mm). T, Urticicola perchtae (SMNS 68657; $\mathrm{H}=3.4 \mathrm{~mm}, \mathrm{D}=4.5 \mathrm{~mm}$ ). U, Discus pleuradrus (SMNS 68655; H = $2.0 \mathrm{~mm}, \mathrm{D}=3.5 \mathrm{~mm}$ ). V, Granaria sp., spire apex (SMNS 101367; H = $1.7 \mathrm{~mm}, \mathrm{D}=1.4 \mathrm{~mm}$ ). W, Gastrocopta acuminata (SMNS 101675; H = $2.6 \mathrm{~mm}$ ). X, Gastrocopta nouletiana (SMNS 1016764; H = $2.1 \mathrm{~mm}$ ). Y, ?Truncatellina sp. (SMNS 101676; H = $0.8 \mathrm{~mm}, \mathrm{D}=0.9 \mathrm{~mm}$ ). Z, Archaeozonites costatus (SMNS $65617 ; \mathrm{D}=25.8 \mathrm{~mm}$ ). 
2016 Gastrocopta nouletiana (Dupuy): Salvador et al., p. 140, figs. 3Q-R.

Material examined. Oggenhausen 2: SMNS 68656 (one spc.), 101674 (ten spc.).

Dimensions. SMNS 1016764: $\mathrm{H}=2.1 \mathrm{~mm}$.

Remarks. The present specimens compare well to Gastrocopta nouletiana, by their slender shell and number and shape of their apertural barriers (totaling seven): suprapalatal tooth, upper palatal tooth, lower palatal tooth, basal tooth, columellar lamella, infraparietal lamella and anguloparietal lamella. The anguloparietal lamella has an overall bifid aspect, with the angular lamella more vertically positioned and the parietal lamella folded towards the outer lip. Gastrocopta nouletiana is common in the European Miocene and shows some variability in the strength of the apertural barriers, with the weaker ones eventually absent (Salvador, 2015).

Family VERTIGINIDAE Fitzinger, 1833

Truncatellina Lowe, 1852

Type species. Pupa linearis Lowe, 1852.

?Truncatellina $\mathrm{sp}$.

(Figure 2Y)

Material examined. Oggenhausen 2: SMNS 101676 (one spc.). Dimensions. SMNS 101676 (fragmentary specimen): $\mathrm{H}=0.8 \mathrm{~mm}, \mathrm{D}=0.9 \mathrm{~mm}$.
Remarks. Only a single spire apex was found. The shell size, overall shape of the whorls and teleoconch sculpture are very reminiscent of Truncatellina, a widespread genus in the European Miocene. However, a more precise identification is impossible.

Superfamily ZONITOIDEA Mörch, 1864 Family ZONITIDAE Mörch, 1864

Archaeozonites Sandberger, 1872

Type species. Archaeozonites subverticillus Sandberger, 1875. Archaeozonites costatus Sandberger, 1875

(Figure 2Z)

1875 Archaeozonites costatus Sandberger, p. 604.

1923 Zonites (Aegopis) costatus (Sandberger): Wenz, p. 254. 1927 Zonites (Aegopis) costatus costatus (Sandberger): Berz \& Jooss, p. 202.

1976 Archaeozonites costatus Sandberger: Schlickum, p. 18, pl. 5, fig. 66.

2002 Miozonites costatus (Sandberger): Binder, p. 168, pl. 1, figs. 14-16, pl. 3, fig. 8, pl. 7, fig. 3, pl. 8, fig. 4.

2006 Aegopis costatus (Sandberger): Kókay, p. 77, pl. 29, figs. 11-12.

2007 Miozonites costatus (Sandberger): Jost et al., p. 312. 2013 Archaeozonites costatus Sandberger: Rasser et al., p. 440. 2014 Miozonites costatus (Sandberger): Harzhauser et al., p. 33, pl. 10, figs. 13-19.

Table 1. Species recorded for each site (Ogg. 1: Oggenhausen 1; Ogg. 2: Oggenhausen 2), alongside their identification in the works of Berz \& Jooss (1927) and Böttcher et al. (2009). A "u" (for unconfirmed) indicates species reported by Berz \& Jooss (1927) for which the original material could not be found.

\begin{tabular}{|c|c|c|c|c|}
\hline \multirow{2}{*}{ Species } & \multicolumn{2}{|c|}{ Site } & \multicolumn{2}{|c|}{ Former species identification } \\
\hline & Ogg. 1 & Ogg. 2 & Berz \& Jooss (1927) & Böttcher et al. (2009) \\
\hline \multicolumn{5}{|l|}{ CAENOGASTROPODA } \\
\hline Pomatias conicus & $\mathrm{x}$ & & Tudorella conica conica & - \\
\hline Tinnyea lauraea & $\mathrm{x}$ & & Melanatria escheri turrita & - \\
\hline \multicolumn{5}{|l|}{ HYGROPHILA } \\
\hline Galba dupuyiana & & $\mathrm{x}$ & - & Galba dupuyiana \\
\hline Gyraulus applanatus & & $\mathrm{x}$ & - & Anisus sp. \\
\hline Lymnaea cf. dilatata & & $\mathrm{x}$ & - & Radix sp. \\
\hline Planorbarius mantelli & & $\mathrm{x}$ & - & Planorbarius cornu \\
\hline \multicolumn{5}{|l|}{ STYLOMMATOPHORA } \\
\hline Archaeozonites sp. & $\mathrm{x}$ & & Zonites (Aegopis) costatus costatus & - \\
\hline Discus pleuradrus & & $\mathrm{x}$ & - & Discus pleuradrus \\
\hline Gastrocopta acuminata & $\mathrm{u}$ & $\mathrm{x}$ & Gastrocopta aff. acuminata acuminata & - \\
\hline Gastrocopta nouletiana & & $\mathrm{x}$ & - & Gastrocopta cf. G. nouletiana \\
\hline Granaria sp. & & $\mathrm{x}$ & - & - \\
\hline Megalotachea silvana & $\mathrm{x}$ & $\mathrm{x}$ & $\begin{array}{c}\text { Cepaea eversa larteti, Cepaea sylvana } \\
\text { sylvana }[\mathrm{sic}]\end{array}$ & Cepaea $\mathrm{sp}$. \\
\hline Palaeotachea renevieri & $\mathrm{x}$ & $\mathrm{x}$ & Cepaea renevieri coniuncta & - \\
\hline Pseudochloritis incrassata & $\mathrm{x}$ & $\mathrm{x}$ & $\begin{array}{l}\text { Tropidomphalus (Pseudochloritis) } \\
\text { incrasstus incrassatus }\end{array}$ & - \\
\hline Triptychia kleini & $\mathrm{u}$ & & Triptychia (Triptychia) grandis & - \\
\hline ?Truncatellina $\mathrm{sp}$ & & $\mathrm{x}$ & - & - \\
\hline Urticicola perchtae & & $\mathrm{x}$ & - & Leucochroopsis sp. \\
\hline
\end{tabular}


2015a Archaeozonites costatus Sandberger: Salvador et al., p. 259 , figs. 3Q-R.

2015b Archaeozonites costatus Sandberger: Salvador et al., p. 206, figs. 2O-P.

Material examined. Oggenhausen 1: SMNS 65617 (1 spc.). Remarks. The single specimen compares well with $A$. costatus by its broad conical spire, flattened protoconch $(\sim 1$ whorl, sculptured by fine riblets that grow coarser towards teleoconch), teleoconch sculptured by numerous strong prosocline ribs, flattened whorl profile and prominent keel on body whorl. The species is known from the Early and Middle Miocene of Central Europe, being rather common in the Silvana-beds of southern Germany (Schlickum, 1976; Kókay, 2006). In its original description, A. costatus was not figured nor properly described (Sandberger, 1875). Thus, Lueger (1981) expressed doubts as to the definition of this species. Nevertheless, the present specimen compares well to later descriptions by Gottschick \& Wenz $(1916,1920)$.

\section{DISCUSSION}

Overall, the Oggenhausen molluscan fauna (Table 1) is rather scarce, presenting more species than several deposits known in the literature (e.g. Wenz, 1923), but being much poorer when compared to the large lakes of the German Miocene, such as Randeck Maar, Sandelzhausen, Riedensheim and Steinheim (e.g. Finger, 1998; Salvador, 2015; Salvador et al., 2015a, 2016). There could be some degree of preservation bias in Oggenhausen 2, since the more extensive and careful collection undertook in this locality did not managed to recover microgastropods, which are very common in other German OSM deposits (e.g. Salvador et al., 2016).

As already remarked by Berz \& Jooss (1927), the snail fauna from Oggenhausen 1 is unusual for its lack of freshwater species, with the exception of Tinnyea lauraea. This fact, when allied to the lack of microgastropods (with the possible exception of Gastrocopta aff. acuminata) and the poor and often fragmentary preservation of the larger species, led Berz \& Jooss (1927) to propose rough transport from terrestrial to freshwater sites. Oggenhausen 2 , on the other hand, shows a much better preservation, and also freshwater taxa (Lymnaea, Galba, Gyraulus and Planorbarius) usually found in other German OSM localities (see also Böttcher et al., 2009).

Böttcher et al. (2009) proposed that the deposits of Oggenhausen 2 could be interpreted as a shallow lake or an oxbow lake formed by the river that deposited the "Oggenhausen-Sande" from Oggenhausen 1, surrounded by lush vegetation. However, the scarce snail fauna found in this deposit do not add much information beyond this. The genera recorded from the sites are not very useful for a paleoecological analysis, because their Recent representatives are not strongly related to any particular environment. Overall, the freshwater snail genera are consistent with slow-flowing or stagnant waters, while the terrestrial Discus and Urticicola are consistent with lusher and more humid vegetation (e.g. Lueger, 1981; Kerney et al., 1983; Welter-Schultes, 2012).

\section{ACKNOWLEDGEMENTS}

We are very grateful to K. Wolf-Schwenninger (SMNS) for the SEM images; and to E. Georgopoulou (NHMW, Vienna), J. Prieto (BSPG, Munich) and an anonymous reviewer for their comments and suggestions. This work was partly supported by a doctoral grant from $\mathrm{CNPq}$ (Conselho Nacional de Desenvolvimento Científico e Tecnológico, Brazil) to R.B.S.

\section{REFERENCES}

Berz, K.C. \& Jooss, C.H. 1927. Über die Altersstellung der tertiären Schichten (Süßwasserablagerungen und bunter Breccia) von Oggenhausen bei Heidenheim a. d. Brenz. Centralblatt für Mineralogie, Geologie, Paläontologie, 1927:193-208.

Binder, H. 2002. Die Land- und Süßwassergastropoden aus dem Karpatium des Korneuburger Beckens (Niederosterreich; Untermiozan). Beiträge zur Paläontologie, 27:161-203.

Binder, H. 2004. Terrestrial, freshwater and brachyhaline Gastropoda from the Lower Miocene deposits of Oberdorf (Styria, Austria). Annalen des Naturhistorischen Museums in Wien, 105:189-229.

Binder, H. 2008. The systematic positions of the genera Pseudochloritis C. Boettger 1909 and Joossia Pfeffer 1929. Archiv für Molluskenkunde, 137:1-27. doi:10.1127/arch. moll/0003-9284/137/167-193

Böttcher, R.; Heizmann, E.P.J.; Rasser, M.W. \& Ziegler, R. 2009. Biostratigraphy and palaeoecology of a Middle Miocene (Karpatian, MN 5) fauna from the northern margin of the North Alpine Foreland Basin (Oggenhausen 2, SW' Germany). Neues Jahrbuch für Geologie und Paläontologie Abhandlungen, 254:237-260. doi:10.1127/0077-7749/2009/0011

Bouchet, P. \& Rocroi, J.P. 2005. Classification and nomenclator of gastropod families. Malacologia, 47:1-397.

Bourguignat, J.R. 1881. Histoire Malacologique de la Colline de Sansan. Annales des hautes Études, Sciences Naturelles, 22:1-175.

Braun, A. 1851. Die fossile Fauna des Mainzer Beckens. In: A.F. Walchner (ed.) Handbuch der Geognosie 2, Walchner, p. 11121140 .

Brongniart, M. 1810. Sur des Terrains qui paraissent avoir étè formes sous l'eau douce. Annales du Muséum d'Histoire Naturelle, 15:357-405.

Dunker, W. 1848. Über die in der Molasse bei Günzburg unfern Ulm vorkommenden Conchylien und Pflanzenreste. Palaeontographica, 1:155-168.

Finger, I. 1998. Gastropoden der kleini-Schichten des Steinheimer Beckens (Miozän, Süddeutschland). Stuttgarter Beiträge zur Naturkunde, 259:1-51.

Fischer, J.-C. 2000. Le malacofaune de Sansan. Mémoires du Muséum National d'Histoire Naturelle, 183:129-154.

Gittenberger, E. 1973. Beiträge zur Kenntnis der Pupillacea III. Chondrininae. Zoologische Verhandelingen, 127:3-267.

Górka, M. 2008. Shell colour pattern in two fossil helicid snails, Tropidomphalus incrassatus (Klein, 1853) and Cepaea sylvestrina Gottschicki Wenz, 1919, from the Middle Miocene of Poland. Acta Geologica Polonica, 58:105-111.

Gottschick, F. \& Wenz, W. 1916. Die Sylvanaschichten von Hohenmemmingen und ihre Fauna. Nachrichtenblatt der Deutschen Malakozoologischen Gesellschaft, 48:17-74. 
Gottschick, F. \& Wenz, W. 1920. Die Land- und Süßwassermollusken des Tertiärbeckensv on Steinheim am Albuch. 3. Fortsetzung. Archiv für Molluskenkunde, 52:33-48.

Harzhauser, M. \& Binder, H. 2004. Synopsis of the Late Miocene mollusc fauna of the classical sections Richardhof and Eichkogel in the Vienna Basin (Austria, Pannonian, MN 9-MN11). Archiv für Molluskenkunde, 133:109-165.

Harzhauser, M. \& Kowalke, T.H. 2002. Sarmatian (late Middle Miocene) Gastropod assemblages of the Central Paratethys. Facies, 46:57-82.

Harzhauser, M.; Kowalke, T. \& Mandic, O. 2002. Late Miocene (Pannonian) Gastropods of Lake Pannon with Special Emphasis on Early Ontogenetic Development. Annalen des Naturhistorischen Museums in Wien, 103:75-141.

Harzhauser, M.; Neubauer, T.A.; Gross, M. \& Binder, H. 2014. The early Middle Miocene mollusc fauna of Lake Rein (Eastern Alps, Austria). Palaeontographica A, 302:1-71.

Heizmann, E. 1983. Die Gattung Cainotherium (Cainotheriidae) im Orleanium und im Astaracium Süddeutschlands. Eclogae Geologicae Helvetiae, 76:781-825.

Höltke, O. \& Rasser, M.W. 2013. The chondrinid land snail Granaria (Stylommatophora: Chondrinidae) in the Miocene of the Alpine Foreland: state of the art and taxonomic reassessment. Neues Jahrbuch für Geologie und Paläontologie, 270:181-194. doi:10.1127/0077-7749/2013/0364

Höltke, O. \& Rasser, M.W. 2015. Pseudochloritis insignis - a peculiar large land-snail from the Miocene of SW Germany: taxonomic status and census of morphologically related forms. Journal of Conchology, 42:1-12.

Jost, J.; Kälin, D.; Schulz-Mirbach, T. \& Reichenbacher, B. 2007. Late Early Miocene lake deposits near Mauensee, central Switzerland: fish fauna (otoliths, teeth), accompanying biota and palaeoecology. Eclogae Geologicae Helveticae, 99:309-326. doi: 10.1007/s00015-006-1198-5

Kadolsky, D. 1995. Stratigraphie und Molluskenfaunen von „Landschneckenkalk“ und „Cerithienschichten“ im Mainzer Becken (Oberoligozän bis Untermiozän?), 2: Revision der aquatischen Mollusken des Landschneckenkalkes. Archiv für Molluskenkunde, 124:1-55.

Kerney, M.P.; Cameron, R.A.D. \& Jungbluth, J.H. 1983. Die Landschnecken Nord- und Mitteleuropas. Hamburg, Verlag Paul Parey, 384 p.

Klein, R. 1846. Conchylien der Süßwasserkalkformation Württembergs. Jahreshefte des Vereins für vaterländische Naturkunde in Württemberg, 2:60-116.

Klein, R. 1853. Conchylien der Süßwasserkalkformation Württembergs. Jahreshefte des Vereins für Vaterländische Naturkunde in Württemberg, 9:203-223.

Kókay, J. 2006. Nonmarine mollusc fauna from the Lower and Middle Miocene, Bakony Mts, W Hungary. Geologica Hungarica, Series Palaeontologica, 56:3-196.

Kowalke, T. 2004. Evolution of the Pachychilidae Toschel, 1857 (Caenogastropoda, Cerithioidea) - from the Tethys to modern tropical rivers. Zitteliana A, 44:41-50.

Kowalke, T. \& Reichenbacher, B. 2005. Early Miocene (Ottnangian) Mollusca of the Western Paratethys - ontogenetic strategies and palaeo-environments. Geobios, 38:609-635. doi:10.1016/j. geobios.2004.03.006

Lueger, J.P. 1981. Die Landschnecken im Pannon und Pont des Wiener Beckens, I. Systematik. II. Fundorte, Stratigraphie, Faunenprovinzen. Denkschriften der Akademie der Wissenschaften, mathematisch-naturwissenschaftliche Klasse, 120:1-124.
Maillard, G. 1892. Monographie des mollusques tertiaires terrestres er fluviatiles de la Suisse. Abhandlung der Schweizer Paläontologische Gesellschaft, 18:1-127.

Manganelli, G. \& Giusti, F. 2000. The gastrocoptids of the Fossil Forest of Dunarobba (Central Italy) and a preliminary revision of the European Tertiary nominal species of Albinula and Vertigopsis (Gastropoda Pulmonata: Gastrocoptidae). Bollettino della Società Paleontologica ltaliana, 39:55-82.

Mathéron, P. 1842. Catalogue méthodique et descriptif des corps organisés fossiles du Département des Bouches-du-Rhône et lieux circonvoisins; précédé d'un mémoire sur les terrains supérieurs au Grès Bigarré du S.E.de la France. Répertoire des Travaux de la Société de Statistique de Marseille, 6:1-269.

Mikuz, V. \& Pavsic, J. 2000. Brotia (Tinnyea) escheri (Brongniart) iz miocenskih plasti pri Tunjicah. Geologija, 43:43-53.

Moser, M.; Niederhöfer, H.J. \& Falkner, G. 2009. Continental molluscs of the fossil site Sandelzhausen (Miocene; Upper Freshwater Molasse from Bavaria) and their value for paleoecological assessment. Paläontologische Zeitschrift, 83:25-54. doi:10.1007/s12542-009-0013-9

Neubauer, T.A.; Kroh, A.; Harzhauser, M.; Georgopoulou, E. \& Mandic, O. 2014. Synopsis of valid species-group taxa for freshwater Gastropoda recorded from the European Neogene. ZooKeys, 435:1-6. doi:10.3897/zookeys. 435.8193

Nordsieck, H. 2014. Annotated check-list of the genera of fossil land snails (Gastropoda: Stylommatophora) of western and central Europe (Cretaceous - Pliocene), with description of new taxa. Archiv für Molluskenkunde, 143:153-185.

Noulet, J.B. 1854. Mémoire sur les coquilles fossiles des terrains d'eau douce du sud-ouest de la France. Paris, Noulet, 127 p.

Pacaud, J.-M. \& Le Renard, J. 1995. Révision des Mollusques Paléogénes du Bassin de Paris. IV - Liste systématique actualisée. Cossmanniana, 3:155-187.

Papp, A. 1953. Die Mollusken fauna des Pannon im Wiener Becken. Mitteilungen der geologischen Gesellschaft in Wien, 44:85-222.

Papp, A. \& Thenius, E. 1953. Vösendorf - ein Lebensbild aus dem Pannon des Wiener Beckens. Mitteilungen der Geologischen Gesellschaft in Wien, 46:1-109.

Rasser, M.W.et al. 2013. The Randeck Maar: palaeoenvironment and habitat differentiation of a Miocene lacustrine system. Palaeogeography, Palaeoclimatology, Palaeoecology, 392:426453. doi:10.1016/j.palaeo.2013.09.025

Reichenbacher, B. 1989. Feinstratigraphische Gliederung der Kirchberger Schichten (Unter-Miozän) an der Typuslokalität Illerkirchberg bei Ulm. Geologica Bavarica, 94:135-177.

Reichenbacher, B. et al. 2013. A new magnetostratigraphic framework for the Lower Miocene (Burdigalian/Ottnangian, Karpatian) in the North Alpine Foreland Basin. Swiss Journal of Geosciences, 106:309-334. doi:10.1007/s00015-013-0142-8

Salvador, R.B. 2013. The fossil pulmonate snails of Sandelzhausen (Early/Middle Miocene, Germany): Succineidae, Testacelloidea and Helicoidea. Zootaxa, 3721:157-171. doi:10.11646/ zootaxa.3721.2.3

Salvador, R.B. 2015. The fossil pulmonate snails of Sandelzhausen (Early/Middle Miocene, Germany): Ellobiidae, Pupilloidea, and Clausilioidea. Paläontologische Zeitschrift, 89:37-50. doi:10.1007/s12542-013-0210-4

Salvador, R.B.; Prieto, J.; Mayr, C. \& Rasser, M.W. 2016. New gastropod assemblages from the Early/Middle Miocene of Riedensheim and Adelschlag-Fasanerie, southern Germany. Neues Jahrbuch fur Geologie und Palaontologie, 279:127-154. doi:10.1127/njgpa/2016/0546 
Salvador, R.B. \& Rasser, M.W. 2014. The fossil pulmonate snails of Sandelzhausen (Early/Middle Miocene, Germany): Hygrophila, Punctoidea and limacoids. Archiv für Molluskenkunde, 143:187-202.

Salvador, R.B.; Rasser, M.W. \& Höltke, O. 2015a. Fossil gastropods from Miocene Lake Randeck Maar and its hinterland (SW Germany). Neues Jahrbuch für Geologie und Paläontologie, 277:251-273. doi:10.1127/njgpa/2015/0505

Salvador, R.B.; Sach, V.J. \& Valentas-Romera, B.L. 2015b. The fossil continental mollusks in the Upper Freshwater Molasse (Middle Miocene) of the districts of Biberach, Ravensburg and Neu-Ulm, Germany. Revista Brasileira de Paleontologia, 18:201-216. doi:10.4072/rbp.2015.2.02

Sandberger, F. 1870-1875. Die Land-und Süßwasserconchylien der Vorwelt. Wiesbaden, Kreidel, 1000 p.

Schlickum, W.R. 1964. Die Molluskenfauna der Süßbrackwassermolasse Niederbayerns. Archiv für Molluskenkunde, 93:1-68.

Schlickum, W.R. 1966. Die Molluskenfauna der Kirchberger Schichten des Jungholzes bei Leipheim/Donau. Archiv für Molluskenkunde, 95:321-335.

Schlickum, W.R. 1970a. Die Molluskenfauna der Kirchberger Schichten des Chiemsee-Gebietes. Geologica Bavarica, 63:143158.

Schlickum, W.R. 1970b. Die Molluskenfauna der oberhelvetischen bis untertortonen brackischen und ausgesüßten Teile der Kohlenbohrungen zwischen Trostberg a. d. Alz und Tittmoning a. d. Salzach. Mitteilungen der Bayerischen Staatssammlung für Paläontologie und historische Geologie, 10:175-188.

Schlickum, W.R. 1976. Die in der pleistozänen Gemeindekiesgrube von Zwiefaltendorf a. d. Donau abgelagerte Molluskenfauna der Silvanaschichten. Archiv für Molluskenkunde, 107:1-31.

Schlosser, M. 1926. Über das geologische Alter der Wirbeltierfauna von Oggenhausen auf der Heidenheimer Alb und über die
Faunen aus dem bayrischen Flinz. Centralblatt für Mineralogie, Geologie und Paläontologie, 1926:198-208.

Schlotheim, E.F. 1820. Die Petrefactenkunde auf ihrem jetzigen Standpunkte durch die Beschreibung seiner Sammlung versteinerter und fossiler Überreste des Thier-und Pflanzenreichs der Vorwelt erläutert. Gotha, Becker'schen Buchhandlung, $437 \mathrm{p}$.

Schnabel, T. 2006. Die känozoischen Filholiidae Wenz 1923. Teil 3: Die miozänen Vertreter der Gattung Triptychia (Gastropoda, Pulmonata, Clausilioidea). Archiv für Molluskenkunde, 135:133203. doi:10.1127/arch.moll/0003-9284/135/133-203

Schütt, H. 1967. Die Landschnecken der untersarmatischen Rissoenschichten von Hollabrunn, N.Ö. Archiv für Molluskenkunde, 96:199-222.

Stworzewicz, E. 1999. Miocene land snails from Belchatów (Central Poland). IV: Pupilloidea (Gastropoda Pulmonata). Systematic, biostratigraphic and palaeoecological studies. Folia Malacologica, 7:133-170. doi:10.12657/folmal.007.015

Thomä, C. 1845. Fossile Conchylien aus den Tertiärschichten bei Hochheim und Wiesbaden gesammelt und im naturhistorischen Museum zu Wiesbaden aufgestellt. Jahrbuch des Nassauischen Vereins für Naturkunde, 2:125-162.

Welter-Schultes, F. 2012. European Non-marine Molluscs, a Guide for Species Identification. Göttingen, Planet Poster Editions, $760 \mathrm{p}$.

Wenz, W. 1923. Gastropoda extramarina tertiaria. In: C. Diener (ed.) Fossilium Catalogus I: Animalia, W. Junk, p. 1-352.

Wenz, W. \& Edlauer, A. 1942. Die Molluskenfauna der oberpontischen Süßwassermergel vom Eichkogel bei Mödling, Wien. Archiv für Molluskenkunde, 74:82-98.

Zöbelein, H.K. 1954. Helvetische Landschnecken aus einem Knollenkalk bei Riedöschingen (Baden). Paläontologische Zeitschrift, 28:155-158.

Received in October, 2015; accepted in March, 2016. 